\title{
A pie de foto
}

José Quintanilla Arquitecto, Pontificia Universidad Católica de Chile.

La observación atenta de la disposición de los objetos dentro del espacio cotidiano de trabajo de un arquitecto y de un artista, permite reflexionar sobre la obra y el legado de Calder y Lewerentz.

Estudio de Calder en RoXbury. NADA de esto es fijo / En Calder es reconocible la intención de hacer sensible el sentido, poner en tensión los sentidos... Y las densidades más altas de sentido yacen en lo inmediato, en lo que está más patentemente a la mano. Calder nos recuerda que cada objeto posee una realidad táctil y que tal circunstancia hace que no podamos desvincular la construcción de la percepción. Parece como si todo se hubiese descolgado del techo, o mejor, en el suelo quedó lo que no tuvo capacidad de vuelo. ¿Qué cosas útiles somos capaces de reconocer en este lugar? ¿Qué cosas somos capaces de catar en este silencio?

Calder nos da a ver... cosas que están a pie de calle, a la mano, que forman parte de la cotidianeidad, del día a día... que nos fuerzan a poner lo extraordinario en la mirada... a través del juego.

ESTUDio DE LEWERENTZ EN LUND. HABITAR CON LO SUFICIENTE / Este es el último lugar de trabajo de un hombre que supo alcanzar lo absoluto con lo suficiente, que buscó una arquitectura en cierto modo esencial (no se entienda minimalista) apoyada en la construcción más que en la imagen.

En medio de esta provisionalidad se recoge una obra de enorme veracidad constructiva, que se caracterizó por el modo radical de plantear las soluciones formales y en la que aparece la asociación estrechísima de
Careful observation of the distribution of objects within a daily work space by an architect and an artist, enables reflection on the work and legacy of Calder and Lewerentz.

rito (vida/acto) y realidad material (dimensión táctil de la arquitectura). Verlo así lleva a entender la arquitectura como medio preciso para una forma de vida.

Este espacio, conocido como la caja negra, constituye el refugio de un hombre que día a día se retira a recoger el trabajo de toda una vida. Es un espacio sin ventanas, un espacio para la concentración, la única comunicación con el exterior son tres lucernarios dispuestos regularmente (tres es el mínimo de elementos para dar la idea de ritmo). Interiormente y en correspondencia con el temperamento de Lewerentz las paredes se pintan de riguroso negro, con un revestimiento de aluminio en el techo para aprovechar la reverberación de la luz natural proveniente del exterior.

Junto a la pared del fondo y sobre unos caballetes se aprecia la maqueta de la iglesia de San Pedro en Klippan, una de sus obras más notables. Los archivadores y paquetes de documentos se presentan ordenados. En primer plano, una mesa despejada. La mirada cae y apreciamos un sencillo suelo de madera.

Pero aquí el trabajo es otro, ¿para qué puede servir ocuparse cada día con uno mismo? ARo

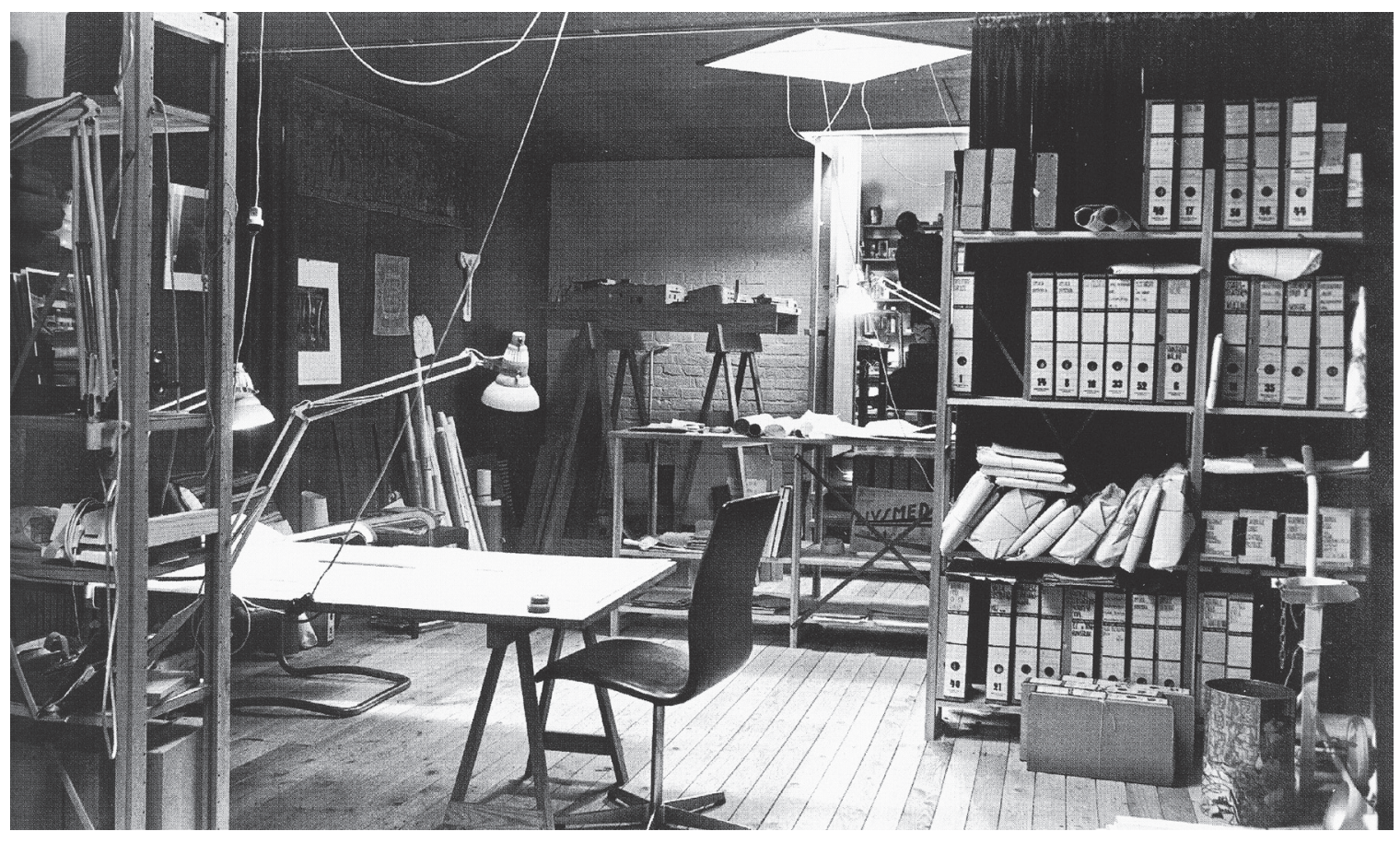


01 Fotografía del estudio del arquitecto sueco Sigurd Lewerentz (1885-1975) extraída del libro Cent maisons pour cent architectes, Taschen, 2004, p. 237. Corresponde al último estudio que va a tener arquitecto en la ciudad de Lund, al sur de Suecia. Fue construido por su amigo y gran admirador Klas Anselm en el jardin de una de sus propiedades.

02 Fotografía de Pedro E. Guerrero del estudio del escultor Alexander Calder (1898-1976) en Roxbury, año 1963. Esta fotografía está extraída del catálogo de la exposición Calder: la gravedad y la gracia organizada por el Museo Guggenheim Bilbao (18 de marzo-7 de octubre de 2003) y coproducida por el Museo Nacional Centro de Arte Reina Sofía, Madrid (18 de noviembre de 2003-18 de febrero de 2004). Tf Editores, Madrid, 2003, p. 40.

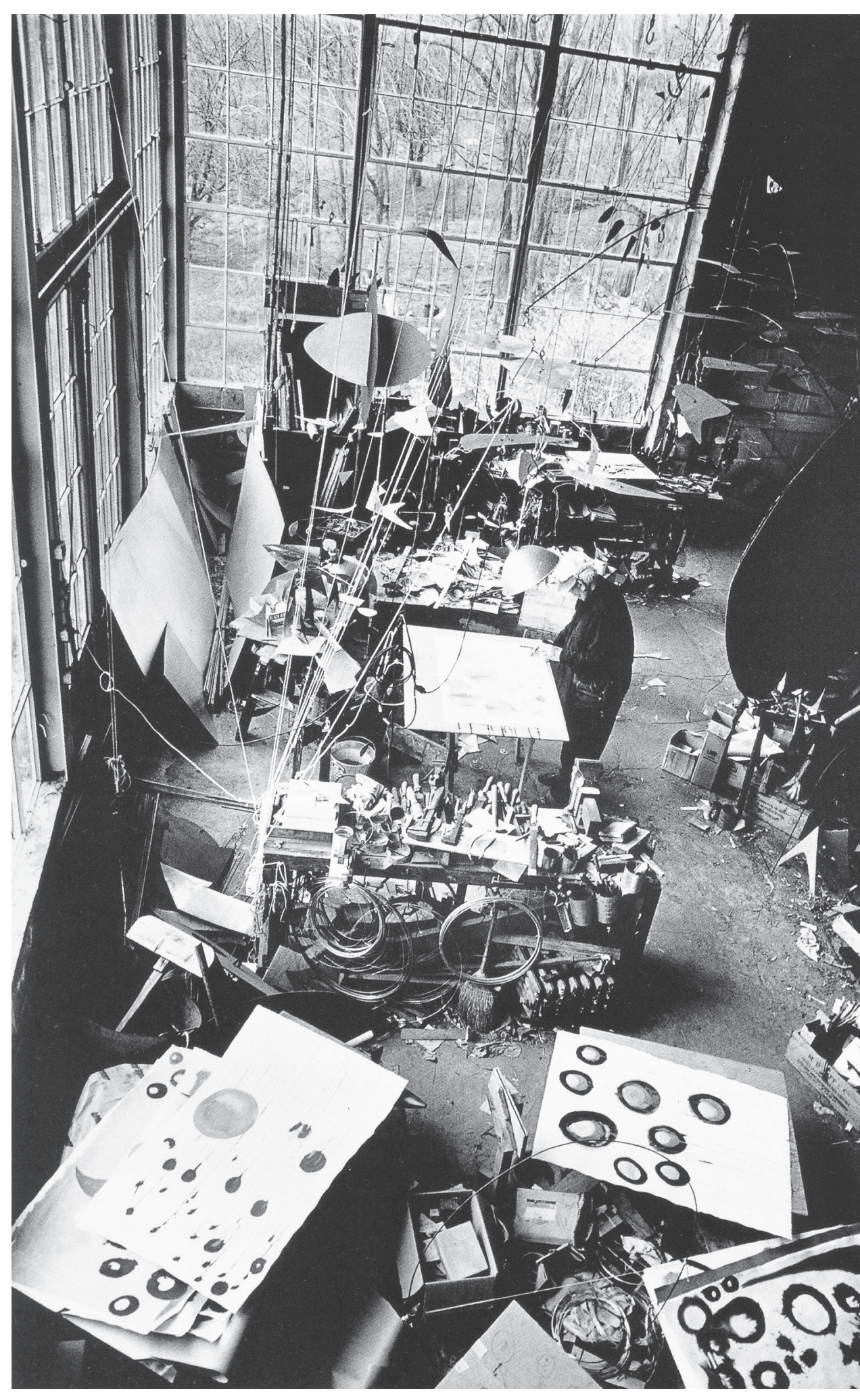

